\title{
A tutorial on some issues and methods in serial pattern research
}

\author{
MARI RIESS JONES \\ Ohio State University, Columbus, Ohio 43210
}

\begin{abstract}
In a review of major trends in serial pattern research, the issue of structural ambiguity and its relationship to representations of hierarchical and nonhierarchical patterns is considered. Also discussed is the role of various kinds of serial rules that can relate events within a sequence (e.g., nominal, ordinal, interval rules), as well as new developments in representing time relationships. Some practical problems that arise in the study of perception of serial structure are shown to involve its formal specification and the enlistment of appropriate controls. Major pitfalls are illustrated using recent research with music-like patterns (e.g., Deutsch, 1980).
\end{abstract}

This paper is about some issues and methodological practices that have developed over the last decade in serial pattern research. It seems important that knowledge that has accumulated in this less visible field be made more accessible to mainstream psychologists, particularly now when stirrings of interest in music perception are evident.

Researchers in serial pattern perception are not overwhelming in number but, perhaps because of renewed concern with musical stimuli, their ranks are gradually increasing. Yet, issues familiar to those of us who have labored in this field for several years remain unfamiliar to newcomers. This is too bad, because a familiarity with some of these issues can engender a healthy awareness of certain pitfalls. Here I present a brief analysis of major trends in serial pattern research with the aim of alerting others to pitfalls in which some of us have floundered. For illustrative purposes, I shall rely upon some recent experiments reported by Deutsch (1980) that involve musiclike serial patterns. These experiments are instructive because, superficially, they appear to support Deutsch's conclusions that people "perceive hierarchical structure" and that rhythm has a "profound effect on perceived structure." In fact, such conclusions are suspect, because these experiments are based upon inadequate manipulations of hierarchical structure and upon inappropriate control conditions.

But let us begin at the beginning with some historical perspective on these matters.

This research was sponsored by Grant BNS-78-06997 awarded to the author by the National Science Foundation. Requests for reprints should be sent to Mari R. Jones, Department of Psychology, Ohio State University, 404-C West Seventeenth Avenue, Columbus, Ohio 43210. The author is indebted to June Baird, Susan Burbidge, John Flach, June Hahn, Walter Johnson, Gary Kidd, Daryle Maser, and Louis Tijerina, and to two anonymous reviewers for helpful comments on an earlier version of this manuscript.

\section{HISTORICAL BACKGROUND}

Simon and Kotovsky (1963) and Simon and Sumner (1968) were among the first to take seriously the problem of rule representation in event sequences. The Thurstone Letter Series Completion task was a favorite here (Kotovsky \& Simon, 1973; Simon \& Kotovsky, 1963). Sequences were letter patterns such as A B M C D M ... which could be extended if one discovered the correct serial rule(s). Typically, these rules reflected a problem-solving strategy of transforming the immediately preceding set of events. Thus, to extend this sequence, one applies a "shift one" rule to two of the most recent group of letters, namely C and D (rather than, e.g., "shift two" to A B ), and then insert " $M$." Rules, later standardized as "next" rules, $\mathrm{N}$, operated on the ordinal alphabet of letters. These rules, like all to follow, operated forward in time so that a rule always changed one event into a "later" event. Later, with Sumner, Simon (Simon \& Sumner, 1968) extended this analysis to musical structure. Here musical scales were treated as circular alphabets, and "next" $\left(\mathrm{N}^{1}\right)$ and "next-of-next" $\left(\mathrm{N}^{2}\right)$ were defined to explain generation of musical fragments such as $\mathrm{C}_{4} \mathrm{D}_{4}$ or $\mathrm{C}_{4} \mathrm{E}_{4}$, respectively (using the $\mathrm{C}$ major scale ${ }^{1}$ ). Some rules were also introduced to represent rhythm; these took the form of strings of time rules.

Hierarchies of rules were first popularized by Frank Restle (Restle, 1970; Restle \& Brown, 1970). Restle emphasized alphabets in which rules had interval properties, and he originally defined a set of rules known as mirror image (M), transposition $\left(\mathrm{T}^{\mathrm{j}}\right)$, and repetition (R). Mostly rules were applied to an alphabet involving a row of six lights (1 233456 ). The $M$ rule involved a complementary transform of, for example, 1 into 6 or 2 into 5 plus a concatenation of the argument with the resultant: $M(1)=16$. For simplicity, I standardize the $\mathrm{M}$ operator to $\mathrm{C}$ for com- 
plement (cf. Simon, 1972). Transposition rules functioned much as Simon's "next" rules, shifting an event $\mathrm{j}$ units along an interval alphabet; I standardize these as $\mathrm{N}^{ \pm j}$ rules. Restle combined these rules in special ways to create patterns with hierarchical structure. Figure la shows a typical example of one of Restle's binary hierarchies.

Regardless of the particular rules involved, a hierarchy was defined as a particular abstract configuration of rules with the main prerequisite that lower level arguments could be recursively rewritten in terms of successively higher level rules. Thus, the sequence in Figure la shows that the fragment 1212 when advanced by $\mathrm{N}$ to 2323 , can be, in turn, operated upon by $\mathrm{C}$ to yield 1212232365655454 . Conventionally, this hierarchical sequence is expressed recursively as $\mathrm{C}(\mathrm{N}(\mathrm{R}(\mathrm{N}(1)))$ ), where the initial event (in this case also the argument, namely 1 ) is often referred to as the reference event.

Hierarchies then involve multiple levels of rules. Originally, evidence for the psychological reality of higher levels of rules came from: (1) profiles of anticipation errors; and (2) relative performance levels with "disjoint" control sequences in which lower level rule chunks were scrambled (Restle \& Brown, 1970). Anticipation error profiles showed that more errors happened at higher level transition points (e.g., $\mathrm{C}, \mathrm{N}$ in Figure 1a) than at lower order transitions (Restle \& Brown, 1970). Because these profiles were orderly, this was evidence that people became sensitive to higher levels of serial structure. Furthermore, overall learning was faster with the hierarchical rule arrangements than with disjoint controls. Since these control patterns exhibited lower order rules that related events within chunks, but they usually failed to display the higher order rules Restle had outlined, Restle and Brown (e.g., 1970) concluded that the presence of these higher level rules facilitated learning.

Often this early research relied heavily upon the serial anticipation paradigm. Jones $(1973,1976 \mathrm{a})$ extended examination of serial structure to serial reconstruction tasks. Some patterns used here involved

a) Hierarchical Rule Structure

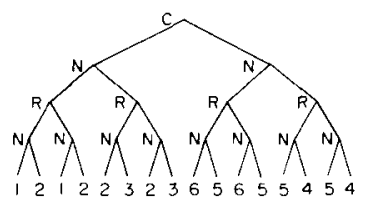

b) Non-Hierarchical Rule Structure (Linear)

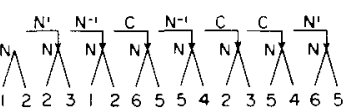

Figure 1. Higher order rule structures using rules from Restle's system: (a) a hierarchical rule tree has multiple rule levels; and (b) a nonhierarchical tree has lower level rule chunks related successively across a single level. (Note that in Restle's system the transpose rule was defined in a special way to include $N \pm 1$ when used with the complement rule, $C$.) ordered digit groups but more recently tone sequences were also studied (e.g., Jones, Maser, \& Kidd, 1978). In reconstruction tasks, clearcut error profiles that reliably reflect higher order rules are harder to come by, although jagged serial recall curves are not at all uncommon. It is simply that inferences based on these profiles are more risky; some of the problems involved here are discussed by Jones (1974). As a result, in pattern reconstruction tasks it becomes quite important to incorporate control sequences which systematically violate higher order rule structure (e.g., Jones, 1976a; Jones \& Baird, 1979).

Throughout this period, the working definition of a hierarchy remained the same. A hierarchical pattern was taken to be one in which: (1) multiple levels of rule structure exist; and (2) symmetrical arrangements of rules across these levels can be demonstrated. Such a pattern can always be expressed recursively. The simplest clue to a hierarchical serial pattern is that the entire second half of the sequence can always be generated directly from the first half by a single rule (e.g., C in Figure 1a). Now, as Simon (1972) has noted, hierarchical patterns represent a very special subset of structural patterns, a subset which involves more than a first-order periodicity (i.e., single level). Not all patterns with higher order structure are hierarchical patterns.

A nonhierarchical pattern with higher order structure is shown in Figure 1b. This pattern involves merely a rearrangement of some of the lower order chunks in Restle's hierarchical sequence:

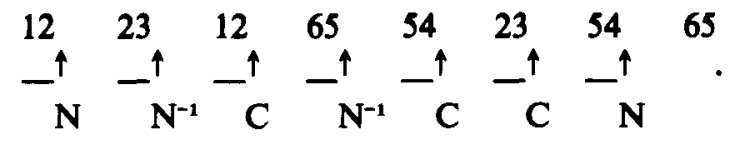

Clearly, there is patternness here. Lower order units preserve a uniform absolute difference (i.e., $\mathbf{N}^{ \pm 1}$ ). Also, at a higher level, rules can be found in Restle's system which transform each chunk into the next (where _ $\uparrow$ now symbolizes an argument transformed without concatenation). This sort of higher order structure was termed a linear structure and was distinguished from hierarchical structure (Jones, 1974; Jones \& Zamostny, 1975). Obviously, as long as rules exist that relate successive chunks, there are many possible linear rule arrangements. A true linear pattern is one based upon an arrangement in which only a single higher order rule level is identifiable. Such a pattern cannot be represented with recursively nested rules.

\section{Structural Ambiguity and Rule Configurations}

The distinction between linear and hierarchical rule representations has broad implications for a major problem in serial pattern research, namely, 
the problem of structural ambiguity (Greeno \& Simon, 1974; Jones, 1974; Restle, 1976). Sometimes a pattern can be formally represented in several different ways. This is especially true for hierarchical patterns. In many cases, a hierarchical pattern can be expressed formally also as a linear sequence of rules: The hierarchical sequence

\section{$12 \quad 234556$,}

for example, recursively is $\mathrm{N}^{3}\left(\mathrm{~N}^{1}\left(\mathrm{~N}^{1}(1)\right)\right)$. This expression reflects the existence of multiple rule levels where higher order rules relate remote events. Thus, $\mathrm{N}^{3}$ transforms the reference event into 4 at the fourth serial location. But this same sequence may also be expressed by a symmetrical linear rule arrangement:

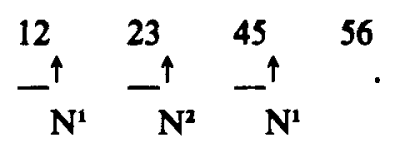

Originally, the inherent ambiguity of hierarchical sequences was not obvious, largely because Restle's rule system was based upon an incomplete rule set. That is, because Restle's rule system did not include certain logical rules within the set he examined, it was difficult to show that there existed several different ways to express the same sequences. Nevertheless, it was possible to show that many true hierarchical patterns were expressible either in terms of overlapping rules or as strings of rules. With true hierarchical patterns, the linear rule representation always involved a symmetrical arrangement of rules such as that shown in Expression 3. Conversely, if the arrangement of rules in a linear rule string is symmetrical, then it can also be expressed recursively as a hierarchy of rules. In each case, the rewriting involves systematic substitution of one rule for two others, or vice versa. (This substitution is best expressed in terms of closure properties of symmetry groups, a topic considered shortly.)

The real problem comes, therefore, in using hierarchical patterns as stimuli from which inferences about perceived higher order structure are drawn. They are ambiguous stimuli. One can often find at least two different rule representations of a hierarchical pattern. Indeed, Greeno and Simon (1974) showed that several formal rule representations can underlie hierarchical patterns. They were interested primarily in reflecting flexibility in output strategies. Thus, given a hierarchical sequence, they showed that it could be produced by a strategy following a linear string of rules (push-down-stack interpreter), by a strategy following overlapping rules (doubling interpreter), or by a strategy based simply upon transforming the initial event (recompute interpreter). Thus, the push-down-stack interpreter reflects a strat- egy in which rules operate primarily upon immediately preceding groups of elements, a feature that is consistent with a linear representation of rules. The doubling interpreter, on the other hand, reflects a strategy which, as Greeno and Simon note, is most consistent with Restle's recursively based hierarchical rule trees.

Greeno and Simon argued that task demands and individual differences determine which production strategy is used, and this choice, in turn, determines pattern difficulty. The same pattern will be easy or hard, depending upon how you are forced to produce it. But the actual identification of which strategy is involved in any given task is a tricky experimental problem, one not addressed by Greeno and Simon.

Although Greeno and Simon assumed that output strategies were independent of encoding, one need not accept this assumption. The issue of structural ambiguity exists for perception as well as for production. That is, given that a hierarchical pattern affords several different rule configurations, which one do people perceive (Jones, 1974; Restle, 1976)? It is possible that people will perceive a pattern only in one of two ways: either as a string of higher order rules at a given level (as in the linear expression) or as overlapping higher level rules that relate remote events across many levels (as in the hierarchical expression). Therefore, if by "hierarchical"' we continue to mean a set of multiple overlapping rules, then this quite simply suggests that hierarchical patterns may not necessarily be perceived as such.

The specter of structural ambiguity hastened development of specialized control sequences, transfer designs, and carefully implanted perturbations of particular pattern parts in serial pattern research.

\section{Structural Ambiguity in Kinds of Pattern Rules}

There is more to this problem of structural ambiguity. Not only may hierarchical patterns be ambiguous with respect to rule configurations, but many structured sequences contain several kinds of pattern rules which usually covary. Jones (1976a, 1978) showed that any pair of events within a sequence may differ nominally, ordinally, or according to interval kinds of alphabetical rules. Thus, the tones $G_{4} B_{4}$ are "different," at the nominal level, but $B_{4}$ is also "higher" (or + ) than $G_{4}$ at the ordinal level. Finally, with respect to the $G$ major scale, $B_{4}$ is $N^{+2}$ units away from $\mathrm{G}_{\mathbf{4}}$. Effectively, this means that any pattern, whether it is linear or hierarchical, can carry several, potentially important, kinds of rule structure. Thus, in addition to having ambiguity with respect to linear and hierarchical combinations of alphabetic rules, the pattern in Expression 2 has the following series of contour relations:

$$
+0++ \text { ot. }
$$


The problem of making inferences about a person's use of particular interval rules therefore is complicated by the fact that ordinal and nominal rules covary with interval rule structure. One solution to this problem involves the use of control patterns having the same contour as experimental patterns (e.g., Dowling \& Fujitani, 1971). That the problem of controlling for contour is a real one is illustrated by work of Massaro and his colleagues (Idson \& Massaro, 1978; Kallman \& Massaro, 1979), who showed that contour alone is a significant cue to melody identification, particularly in forced-choice tasks.

\section{Recent Advances}

Other advances in serial pattern research have centered about two issues: (1) developing a more powerful and coherent rule system for formal representation of pattern structure; and (2) developing a psychological theory to explain how people perceive this structure. Both have implications for the issue of structural ambiguity.

With respect to rule systems, a major advance came in 1974 when, simultaneously, Greeno and Simon (1974) and Jones (1974) discovered the application of group theoretic principles to serial patterns. Because one property of a group of elements is that two rules in the group can always be rewritten in terms of a third rule, these formalisms allowed a clear expression of the ambiguity problem as it related to hierarchical patterns. Restle's rules did not form a complete set, and so they lacked this important group property called closure. For this reason, it was often difficult to demonstrate structural ambiguity within Restle's system. But with the discovery of rule groups, it was possible not only to clarify the issue of structural ambiguity, but also to consider significant higher order structures beyond the special set of hierarchical configurations. In short, groups of symmetry rules broadened the formal basis for identifying psychologically simple patterns.

Abstract representations of two groups of rules, as they apply to musical alphabets, are shown in Figure 2. One is called the "four" group (it represents the Klein four symmetry group) and it contains four rules, an identity rule, I, a complement rule, $\mathrm{C}$, a transpose rule, $\mathrm{Tr}$, and a reflection rule, $\mathbf{R} \boldsymbol{l}$. These rules are most easily defined by the mappings shown in Figure 2a. Here the similarity of complement and reflection rules to flips about imaginary horizontal and vertical axes of the scale, respectively, becomes apparent. The transpose rule functions as a flip about diagonal axes.

The second group, shown in Figure 2b, is referred to as the "next" group and is here defined on the modulus of 7 . This group contains the adding and subtracting rules, $\mathrm{N}^{ \pm \mathrm{j}}$, which can be spatially portrayed as clockwise and counterclockwise rotations on a diatonic circle of modulus 7 , as shown. The modulus of 7 reflects octave equivalence. Octave equivalence means that corresponding scale notes in all octaves can be collapsed onto a unit circle, hence subscripts such as $C_{4}$ and $C_{3}$ do not necessarily distinguish different notes (e.g., $\mathbf{N}^{8}=\mathrm{N}^{1}$ under modulus 7). ${ }^{2}$

Groups of rules such as these have some mathematical properties which make them good candidates for the representation of prototypical symmetries in music. They also facilitate, via the closure property, recursive rewriting of linear rule strings, and vice versa. To illustrate with the "four" group rules of Figure 2, a linear rule string, beginning with the argument $\mathrm{C}_{4} \mathrm{E}_{4}$, yields:

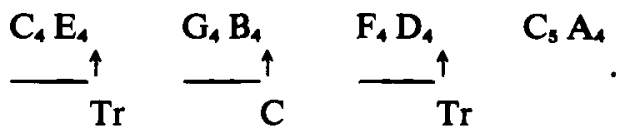

Since the rules form a group, a combination (here a succession operation) of any two always yields another rule in the group, given the closure property. Thus, the transpose rule, $\mathrm{Tr}$, followed by complement, $\mathrm{C}$, always yields reflection, $\mathrm{R} \ell$. Consequently, this pattern is also expressed hierarchically in a recursive formula as $\operatorname{R} \ell\left(\operatorname{Tr}\left(C_{4} E_{4}\right)\right)$. Thus, the flexibility of expressing a symmetrical linear string as a hierarchical rule formulation, or vice versa, depends upon the group's closure property.

Group closure which enters into structural ambiguity can be further illustrated by a common musical example. In the musical sequence $\mathrm{C}_{4} \mathrm{E}_{4} \mathrm{G}_{4}$, the interval between $\mathrm{C}_{4}$ and $\mathrm{G}_{4}$ can be expressed as a compound interval based on a major third (i.e., from $C_{4}$ to $E_{4}$ ) and a minor third (i.e., from $E_{4}$ to $G_{4}$ ), or it can be expressed directly as the combination of these two intervals, namely a perfect fifth (i.e., from $\mathrm{C}_{4}$ to $\mathrm{G}_{4}$ ). The closure property that any two rules in a group result in a third rule that is also in the group describes this state of affairs. In short, group properties formalize and so, in a sense, explain structural ambiguity, but they do not solve the problem.

The rule groups of Figure 2 are presented merely to illustrate two different groups of rules. Here the "next" rules are identified with respect to some modulus (i.e., a numerical base such as 7 for a diatonic scale or 12 for the chromatic scale) and inverses (i.e., $\mathrm{N}^{-j}$ as the inverse of $\mathrm{N}^{+j}$ ) so they have group properties. More extensive groups of rules, which arise from, for example, the chromatic scale, can powerfully capture many significant musical relationships involving diatonic scales, triads, and cadences (see Hahn \& Jones, 1981; Balzano, Note 1). In fact, 
a)

The Four Group

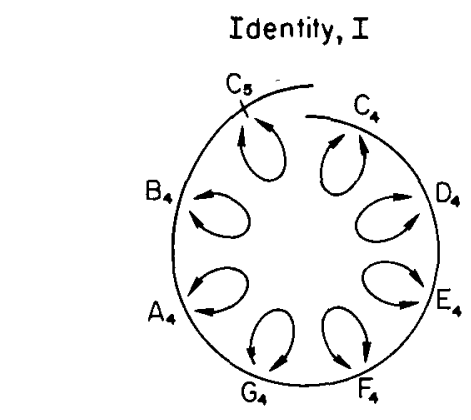

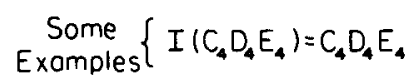
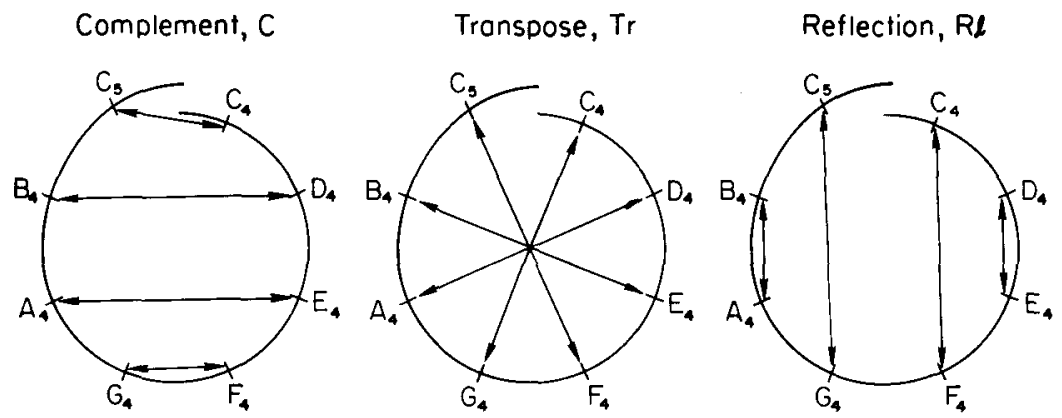

$C\left(C_{4} D_{4} E_{4}\right)=C_{5} B_{4} A_{4}$

$\operatorname{Tr}\left(\mathrm{C}_{4} \mathrm{D}_{4} \mathrm{E}_{4}\right)=\mathrm{G}_{4} \mathrm{~A}_{4} \mathrm{~B}_{4}$

$R \ell\left(C_{4} D_{4} E_{4}\right)=F_{4} E_{4} D_{4}$

b)

The Next Group

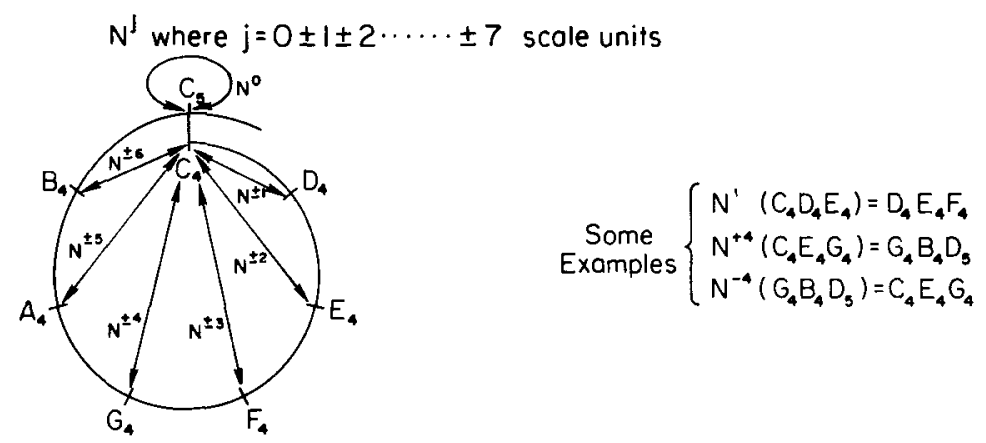

Figure 2. Two different groups of rules applied to a musical (diatonic) alphabet: (a) the "four" group and (b) the "next" group both use transformations involving scale units. (The "next" rules are defined here on a modulus of seven, hence note that subscripts, technically, are meaningless.)

Hahn and Jones (1981) recently expressed many of these relationships formally in the symmetry of a natural growth pattern, the logarithmic spiral. Much remains to be explored, however. For example, we do not yet know how all these rule groups fit together neatly.

Although group properties allow us to rewrite a combination of two rules as a single overlapping rule, this does not mean that any linear string of rules will be automatically converted to a hierarchical one. True linear patterns remain nonhierarchical. Nevertheless, group properties often explain other salient features which may unfold in patterns with other kinds of higher order structure. Consider the sequence ${ }^{3}$ :

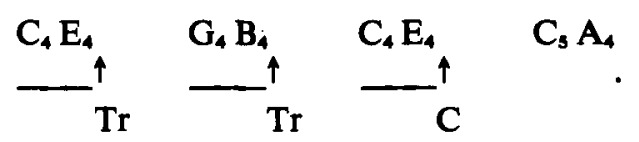

This remains a truly linear pattern. Why? Because, as a nonrepeating pattern, the rules are not symmetrically distributed and, hence, cannot be recursively rewritten. However, its repeated chunk $\left(C_{4} E_{4}\right)$ can be explained through group properties: a transpose rule, $\mathrm{Tr}$, followed by itself, will always yield an identity rule.

Comparing the patterns of Expressions 4 and 5, the reader may well wonder what hierarchical parsimony "buys" for the former. In fact, one is tempted to ask, "Are hierarchical sequences always easier 
than nonhierarchical ones?" The answer is "No." Many things enter in to qualify one's intuitive belief that hierarchical sequences are easier than nonhierarchical ones. The particular interval rules involved and their frequency matter, but so also do nominal (i.e., repeated elements) and ordinal (i.e., contour invariance) rule properties (Jones, 1976a; Jones, Maser, \& Kidd, 1978; Jones \& Zamostny, 1975; Restle, 1976; Restle \& Brown, 1970). But even so, if such variables are all held constant, does one configuration (either linear or hierarchical) prove to be simpler? Unfortunately, we do not know this, for it is very difficult to hold all of these variables still in order to compare a hierarchical arrangement with a linear one.

A second advance came in theory development. Jones (1976b) extended the idea of symmetry rule groups to temporal relationships. ${ }^{4}$ This approach differs from the earlier work of Simon and Sumner in that rhythm can be conceived either in terms of linear string-of-time rules or in terms of overlapping rules whose relative time properties contribute to an accent-rhythm hierarchy.

To illustrate, imagine various durations in a 12-tone sequence with pauses of $600 \mathrm{msec}$ after every third tone. If tones last $300 \mathrm{msec}$, and $150 \mathrm{msec}$ separates neighbors with a three-tone group, then the time structure is as shown in Figure 3 (where stipulations indicate tone durations). Let $\mathrm{T}^{1}$ be a unit time rule of $150 \mathrm{msec}$, then, across the entire sequence, tone onsets are given by the linear string of rules: $T^{3} T^{3}$ $T^{3} \ldots$ Notice that this linear representation can be rewritten hierarchically with overlapping time rules. This hierarchy emphasizes the fact that every fourth note is accented, thus forcing into prominence higher order subsequences called accent subsequences (e.g., accents 1 and 2 for $\mathrm{T}^{24}$; accents $1,3,2,4$ for $\mathrm{T}^{12}$ ). Of course, different time patterns will result in different tones entering into accent subsequences (see also Martin, 1972).

The pattern of Figure 3 illustrates that structural ambiguity is also true of time rules. The sequence can be expressed in lower order periods by $\mathrm{T}^{1}$ or $\mathrm{T}^{3}$, or in terms of higher order periods by $\mathrm{T}^{6}$ or $\mathrm{T}^{12}$. Indeed, different kinds of rhythmic hierarchies can be specified, depending upon the time levels selected. This sort of temporal structure ambiguity underlies some of the differences between Martin's (1972) rhythmic analysis and Povel's (1981). Martin's time hierarchies are built upon multiples of a sequence's smallest time interval (e.g., $T^{1}$ ), whereas Povel has suggested that people may actually select some higher order time period (e.g., $\mathrm{T}^{9}$ ) and subdivide.

Paradoxically, at a more general level there is real value in identifying structural ambiguity. From a theoretical perspective, the ambiguity inherent in these

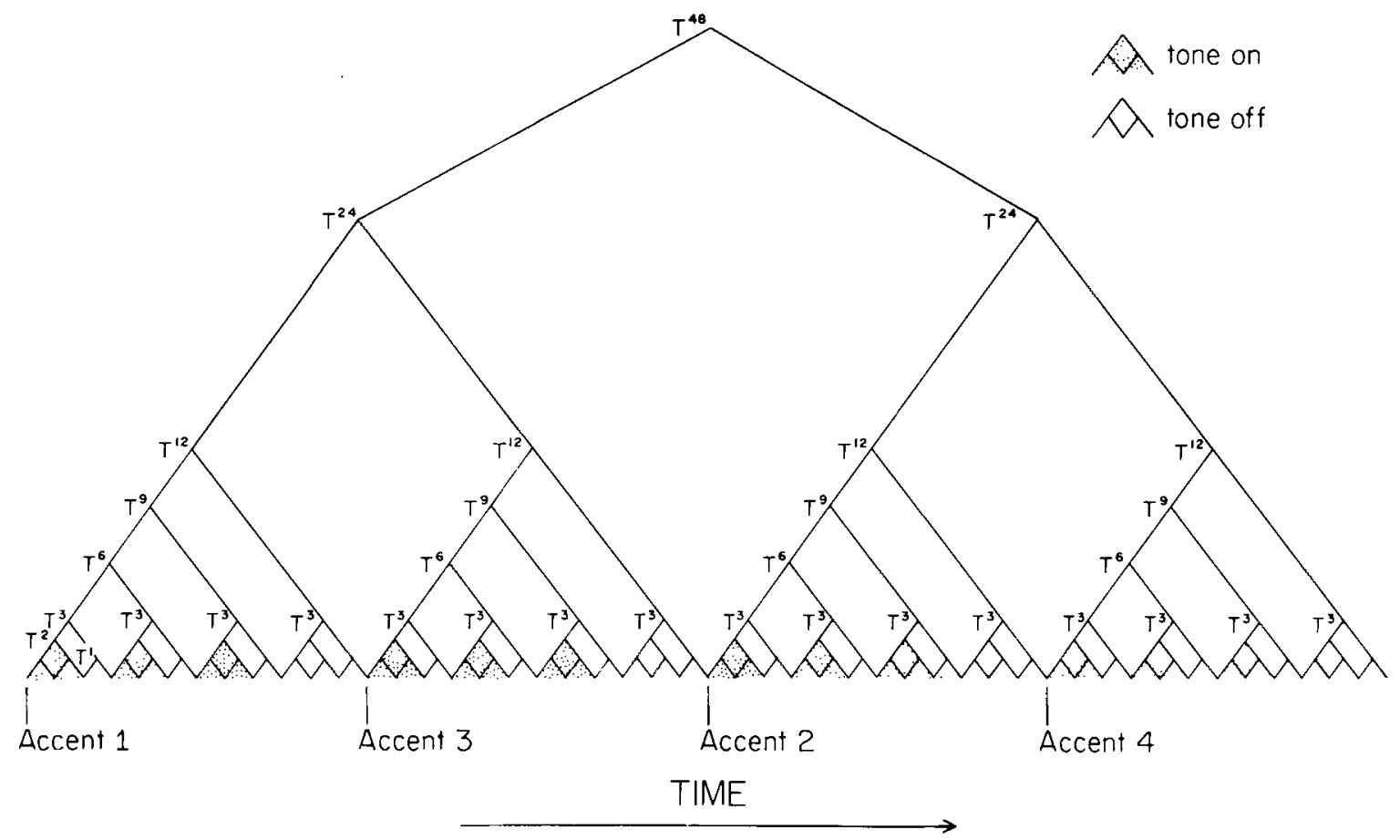

Figure 3. Accent-rhythm hierarchy of time rules for a 12-tone pattern. Notice that if on-times are 300 msec and intertone off-times are $150 \mathrm{msec}$ or $600 \mathrm{msec}$, then the rhythm is easily specified by ratios of time rules: $T^{9} / T^{3}=3$ and $T^{12} / T^{3}=4$, and so forth. 
different descriptions of a temporal sequence cloaks a vehicle for flexible attentional focusing! Jones (1976b, 1981) argued that attention is fundamentally rhythmic and that it follows subjective time rules. This means that attention can be focused upon relationships between neighboring events by synchronizing appropriate subjective time rules with the corresponding lower level temporal periods within a pattern such as $\mathrm{T}^{3}$. Conversely, attention may lock into a higher order period such as $\mathrm{T}^{12}$. Because $T^{12}$ is a multiple of $T^{3}$, the pattern's time structure is ambiguous, and so it affords the shifting of attention from lower to higher periods, or vice versa. A bottom-up attentional strategy involves heightening subjective rhythms corresponding to $\mathrm{T}^{3}$ initially and then expanding to $\mathrm{T}^{12}$. A top-down attentional strategy involves locking into a higher period such as $T^{12}$ first and then contracting to pick up finer details at the level of $\mathrm{T}^{3}$. Obviously these strategy differences conjure up different rhythmic models, models which separately appear to emphasize an individual's use of one or another temporal level within a sequence (e.g., Martin, 1972; Povel, 1981).

Finally, how do these strategies affect one's perception of the melodic pattern? If perception of a given melodic rule depends upon the corresponding temporal level of attending, then a top-down strategy may facilitate pickup of hierarchical arrangements, while a bottom-up strategy may facilitate pickup of linear rule strings.

An important task for future research involves the discovery of ways to measure and evaluate such attentional strategies. We need to uncover variables which stimulate one or the other strategy. In this respect, clearly the objective rhythm will matter. And because rhythm cannot be separated from the temporal placement of accents, a pattern's contour and, for example, melodic rule shifts will also matter. For example, regularly spaced pauses may facilitate heightened attention to higher order periods. Similarly, sequences with regularly spaced directional changes (i.e., accents) may facilitate a top-down strategy wherein attention is targeted over the higher level time periods between contour changes. An interaction of rhythm with contour is suggested by this analysis. Its delineation is a delicate and challenging research problem.

To summarize, serial pattern theory and research distinguishes at least two kinds of higher order structure: hierarchical and linear. The former involves multiple levels of rules that are recursively expressed and the latter involves at most two levels of rules. Often, hierarchical patterns are structurally ambiguous, for they can be reexpressed as linear rule arrangements. Although some patterns expressed as linear rule strings can be also expressed recursively, this is not the case with nonsymmetrical rule arrangements (true linear patterns). Complicating the study of perception of higher order structure is the fact that hierarchical patterns may not always be easier than nonhierarchical ones.

A formal summary of structural ambiguity comes with the representation of serial relationships in terms of groups of rules. When these rules are extended to include linear and hierarchical representations of time periods within sequences, a basis is laid for a dynamic theory of attention. Subjective time rules define a rhythmical attentional process which moves flexibly between pattern levels guided by objective rhythmic and contour features.

\section{PRACTICAL APPLICATIONS AND PITFALLS}

Against this backdrop, let us consider inferences that are permissible with data collected in a serial pattern reconstruction task. Several pitfalls that attend the practical application of serial rule systems in this setting are dramatized by Deutsch's recent studies (Deutsch, 1980).

Deutsch used rules originally introduced by Simon and Sumner (1968) to generate nonrepeating auditory patterns involving 12 pure sine tones. In the first of two experiments, there were four structured sequences and four control sequences. The structured sequences were taken to be hierarchical patterns based upon three-tone arguments, or chunks, and each control sequence was a random rearrangement of the individual tones in a given structured sequence. These eight patterns are shown in Figure 4. In the second experiment, structured sequences had melodic chunks of either three or four tones. Rhythm was also varied. Sequences in Experiment 1 had either no pauses or pauses which divided them into temporal groups of three tones (compatible rhythm) or four tones (incompatible). Sequences in Experiment 2 were divided temporally into groups of two, three, or four tones, thus also allowing compatible vs. incompatible comparisons.

Deutsch found that, with musically sophisticated subjects, serial reconstruction was better with structured sequences than with control sequences. She concluded that "listeners perceive hierarchical structures that are present in tonal sequences." Furthermore, she found that recall was best when temporal segmentation was compatible with melodic chunking. From this she concluded that "temporal segmentation has a profound effect upon perceived structure." She argued that when temporal grouping conflicts with melodic chunks, the perceived rules are more complex.

To evaluate these inferences, it is necessary to examine, first, Deutsch's formal terminology and, second, her methodology.

\section{Deutsch's Terminology and Rule System}

One hopes that science progresses logically by building upon established conventions (cf. Tulving, 1979). One problem in coming to grips with Deutsch's 

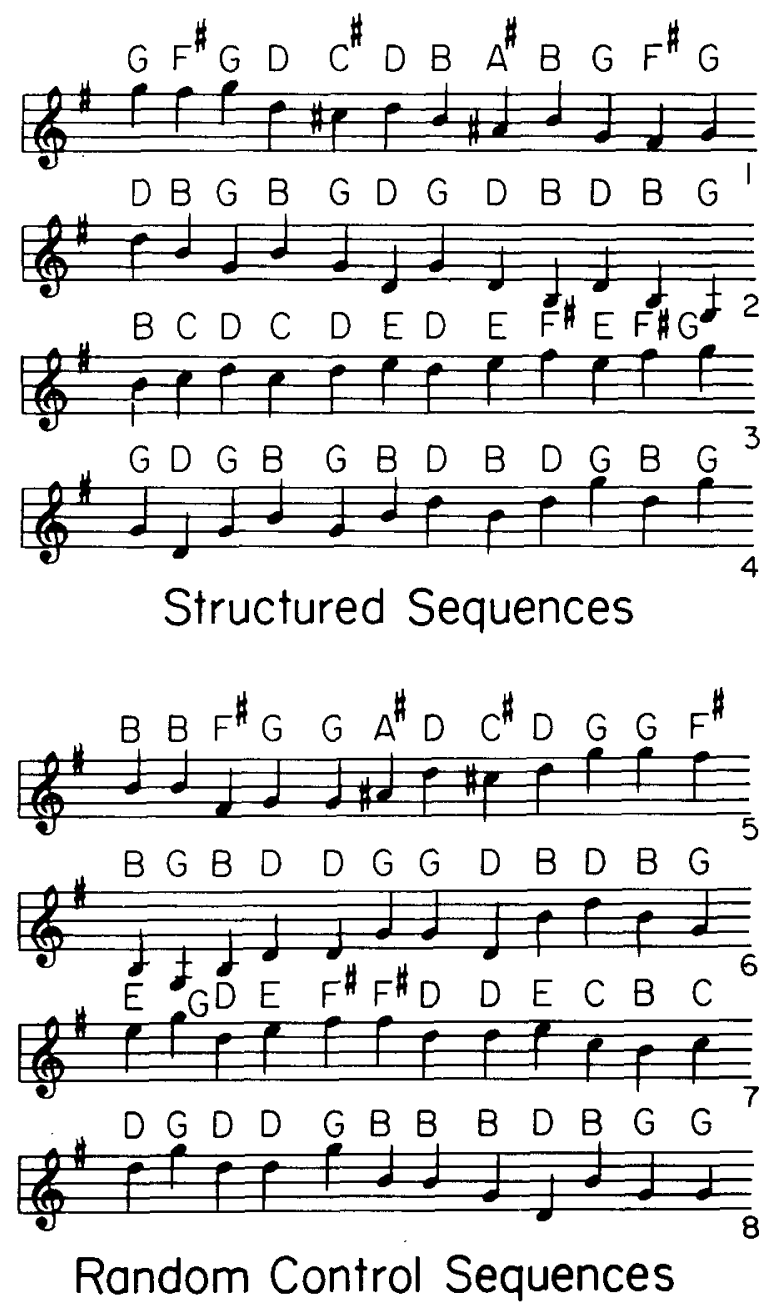

Figure 4. A reproduction, with notated musical labels, of eight sequences (see right-hand enumeration) from Experiment 1 of Deutsch (1980).

experiments is that the sequences used derive from a rule system that is difficult to square with established convention. This becomes apparent in considering Deutsch's use of the term "hierarchical structure." It is not at all clear that what she intends by this term reflects the conventional meaning of the word "hierarchical." As will be shown, Deutsch apparently means not a particular rule configuration, but a twolevel "hierarchy of subsequences." While there is nothing inherently wrong with introducing new meanings for old terms, it is important to be quite clear about it. Deutsch is not. Furthermore, other problems arise in this rule system and the resulting patterns, and together these render conclusions about what a listener actually perceives difficult.

The critical device that Deutsch introduces to achieve hierarchical structure involves two alphabets, one from which melodic rules within chunks are gen- erated and one from which melodic rules between chunks are generated. The former alphabet is denoted $\alpha_{2}$ and the latter, $\alpha_{1}$. Together, pairs of alphabets generate a pattern. In some cases, $\alpha_{1}$ can be the chromatic scale $\left(C_{r}\right)$ and $\alpha_{2}$ a major triad (e.g., $G_{t r}$ for $\mathrm{G}$ major triad), and in other cases $\alpha_{1}=\alpha_{2}$ and a single diatonic scale (e.g., $G$ major) is involved. Table 1 summarizes the alphabet pairs for structured sequences in Deutsch's first study.

A structured pattern then is represented in terms of two alphabetically determined melodic subsequences. For example, Sequence 1 (Figure 4) is developed from rules applied to the $G$ major triad alphabet (i.e., $\alpha_{1}=\mathrm{G}_{\mathrm{tr}}$ ) to give the higher order subsequence of notes.

$$
\text { G..D..B.G... }
$$

A lower order subsequence then comes from different rules applied to the chromatic alphabet (i.e., $\alpha_{2}=C_{\mathrm{r}}$ ), and this dictates that after each of above notes one moves a semitone up and then down. To use Deutsch's example, Sequence 1 (Figure 4) has the higher level rule representation of $3 \mathrm{~N}^{-1}$ for $\alpha_{1}=\mathrm{G}_{\mathrm{tr}}$, and this is combined with the lower level rule representation (for notes within chunks) of $\mathrm{N}^{-1} \mathrm{~N}^{+1}$ for $a_{2}=C_{r}$ to explain:

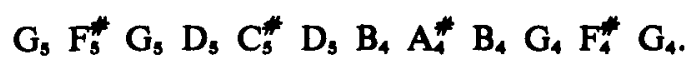

Rules are always specific to an alphabet so that in terms of semitone units, an $\mathrm{N}^{+1}$ rule means one thing in the $G$ major triad alphabet and another in the chromatic scale. The most important consequence of this in Deutsch's two-alphabet system is that rules for lower order chunks cannot always be recursively rewritten in terms of higher order rules. This is true, for example, with Sequence 1 (Expression 7). Thus, over a whole pattern, the usual test for a hierarchical sequence may fail, since the last half of a structured sequence will not necessarily result from the first half by a single transformation.

Deutsch, however, maintains that there is something hierarchical in these structured sequences. So let us focus simply upon the rule configurations that relate notes from higher sequences (as in, e.g., Expression 6). Table 1 summarizes these rules both in terms of Deutsch's encoded representations and as hierarchical (i.e., recursive) expressions. (I have standardized Deutsch's notation to conform to current usage of $\mathrm{N}^{ \pm j}$ rules.) Notice that, at this level, rules which relate only higher order notes in a linear fashion can be recast in terms of a hierarchical formula. Four related points need to be made about these representations: (1) Formally, these patterns are structurally ambiguous with respect to hierarchical vs. linear rule 
Table 1

Formal Representations of Two-Alphabet Sequences

\begin{tabular}{|c|c|c|c|c|c|c|}
\hline \multirow{2}{*}{$\begin{array}{l}\text { Structured } \\
\text { Sequence* }\end{array}$} & \multicolumn{2}{|c|}{ Alphabet Pair } & \multicolumn{2}{|c|}{ Encoded Representations } & \multicolumn{2}{|c|}{ Higher-Order Subsequences } \\
\hline & $\alpha_{1}$ & $\alpha_{2}$ & Deutsch's & Hierarchical & First Note/Chunk & Last Note/Chunk \\
\hline $\begin{array}{l}1 \\
2 \\
3 \\
4\end{array}$ & $\begin{array}{l}G_{t r}\left(G_{4} B_{4} D_{5} G_{5}\right) \\
G_{t r}\left(G_{3} B_{3} D_{4} G_{4}\right) \\
G\left(G_{4} \ldots F_{3}^{*} G_{3}\right) \\
G_{t r}\left(G_{4} B_{4} D_{5} G_{5}\right)\end{array}$ & $\begin{array}{l}\mathrm{C}_{\mathrm{r}}\left(\mathrm{G}_{4} \mathrm{G}_{4}^{\#} \ldots \mathrm{F}_{5}^{\#} \mathrm{G}_{5}\right) \\
\mathrm{G}_{\mathrm{tr}}\left(\mathrm{G}_{3} \mathrm{~B}_{3} \mathrm{D}_{4} \mathrm{G}_{4}\right) \\
\mathrm{G}^{\mathrm{G}}\left(\mathrm{G}_{4} \ldots \mathrm{F}_{3} \mathrm{G}_{5}\right) \\
\mathrm{G}_{\mathrm{tr}}\left(\mathrm{G}_{4} \mathrm{~B}_{4} \mathrm{D}_{5} \mathrm{G}_{5}\right)\end{array}$ & $\begin{array}{l}3 N^{-1} \\
3 N^{-1} \\
3 N^{1} \\
3 N^{1}\end{array}$ & $\begin{array}{l}\mathbf{N}^{-2}\left(\mathbf{N}^{-1}(\mathbf{X})\right) \\
\mathbf{N}^{-2}\left(\mathbf{N}^{-1}(\mathbf{X})\right) \\
\mathbf{N}^{2}\left(\mathbf{N}^{1}(\mathbf{X})\right) \\
\mathbf{N}^{2}\left(\mathbf{N}^{1}(\mathbf{X})\right)\end{array}$ & $\begin{array}{l}G_{5}, \mathrm{D}_{5}, \mathrm{~B}_{4}, \mathrm{G}_{4} \\
\mathrm{D}_{5}, \mathrm{~B}_{4}, \mathrm{G}_{4}, \mathrm{D}_{4} \\
\mathrm{~B}_{4}, \mathrm{C}_{5}, \mathrm{D}_{5}, \mathrm{E}_{5} \\
G_{4}, \mathrm{~B}_{4}, \mathrm{D}_{5}, \mathrm{G}_{5}\end{array}$ & $\begin{array}{l}\mathrm{G}_{3}, \mathrm{D}_{3}, \mathrm{~B}_{4}, \mathrm{G}_{4} \\
G_{4}, \mathrm{D}_{4}, \mathrm{~B}_{3}, \mathrm{G}_{3} \\
D_{3}, \mathrm{E}_{4}, \mathrm{~F}_{3}, \mathrm{G}_{8} \\
\mathrm{G}_{4}, \mathrm{~B}_{4}, \mathrm{D}_{3}, \mathrm{G}_{8}\end{array}$ \\
\hline
\end{tabular}

Note $-G_{t r}=G$ major triad; $G=G$ major scale; $C_{r}=$ chromatic scale. Deutsch's reference event for higher order subsequences is given in italics. $\quad$ *From Figure 4 .

features; (2) Deutsch's rule representation in each case reflects a linear encoding strategy; (3) hierarchical expressions necessarily engage rules and a strategy that are different from those Deutsch has specified; and (4) all four pattern representations reflect essentially equivalent and very restricted higher order rule configurations based upon the $\mathrm{N}^{+1}$ rule and its inverse, and therefore these stimulus patterns offer little generality.

The issue of structural ambiguity has already been discussed. The fact that it exists in Deutsch's pattern means that inferences about perceived structure will be difficult to make without numerous safeguards. I return to this point in the methodology section. The second point concerns Deutsch's use of a linearly encoded representation based on a string of three $\mathrm{N}^{1}$ (or $\mathrm{N}^{-1}$ ) rules. This is especially puzzling in light of Deutsch's conclusions about hierarchical encoding. It is one factor which suggests that by "hierarchy" Deutsch must mean simply a two-tiered sequence. The third point reflects the fact that if people encode hierarchically, rules will be involved that stretch over higher order periods, and, indeed, these rules may be differentially affected by rhythmic formatting. And finally, Deutsch's patterns lack generality. When represented linearly, they repeatedly engage the same rule, and significantly it is an especially simple rule in this case $\left(\mathrm{N}^{+1}\right.$ or its inverse). Because these stimulus patterns are based only upon the $\mathrm{N}^{ \pm 1}$ rule, it is difficult to determine if performance is indeed affected by a particular arrangement of rules or merely by the fact that an especially simple rule is involved. To this point, there is evidence that in nonrepeating serial patterns, serial reconstruction performance does vary greatly with the nature and frequency of particular higher order rules involved (Jones, 1976a; Jones \& Zamostny, 1975). These factors significantly qualify the generality of any findings with respect to structural differences. In short, Deutsch confounds rule configuration with the individual rules used.

Other problems, some with important theoretical implications that cannot be considered here, concern the role of the reference event in Deutsch's rule system. However, I must remark on these because it is easy to misunderstand this part of Deutsch's system and so be confused by it and by the summary of it in Table 1. Briefly, in an apparent attempt to reflect the special role of tonic and dominant notes in music, Deutsch allows a subsequence of rules to begin with any note within a chunk. Thus, in Sequence 2 of Figure 4 , the reference event is not the initial note, but rather the third note, $G_{4}$. Unfortunately, the result of this is (1) even greater structural ambiguity for these sequences, and (2) the introduction of rules that operate backward in time with no clear guidelines for their operation. Thus, in Table 1, the last two columns show that higher order subsequences might begin on (for example) either the first or last note of each opening chunk, and apparently only musical intuition guides these choices. That is not enough.

To summarize, Deutsch has not clarified her meaning of the term "hierarchy." Moreover, her patterns, at one level, can be formally expressed in several ways, not all of which reflect "perceived hierarchical structure." Finally, the patterns selected for study conform to a highly restricted set based primarily upon one rule. This not only confounds rule configuration with rule type but greatly limits the generality of her findings.

\section{Methodological Considerations}

Methodological concerns cannot be separated from theoretical questions. In particular, control sequences in this kind of research must always be carefully constructed so as to convincingly eliminate plausible alternative answers to these questions. While randomly constructed sequences are adequate for some purposes, frequently they are too crude as controls for questions about higher order rule structure. In the present case, I will illustrate this with respect to two questions relevant to Deutsch's studies: (1) "Do people perceive hierarchical structure?" and (2) "Does rhythm affect perception of rule structure (hierarchical or other)?'”

(1) Do people perceive hierarchical structure? We have seen that hierarchical patterns are ones with multiple rule levels and a symmetrical rule configu- 
ration. Furthermore, because they are formally ambiguous, it may be difficult to discover whether a listener encodes a sequence in terms of a string of rules at a single level or in terms of the overlapping rules at multiple levels. It is especially difficult with simple reconstruction tasks in which significant serial intrusion errors are difficult to assess (cf. Restle, 1976).

However, to begin to answer this question, we must first consider ways to demonstrate the psychological reality of multiple levels of structure. If, for example, structural sequences have multiple rule levels and control sequences have no rule levels, lacking even relationships between chunks, then the fact that structured sequences are easier than unstructured ones tells us little about a person's perception of hierarchical structure. To be sure, it tells us that people find some structure easy and randomness difficult, but it does not tell us that people perceive hierarchical structure.

Other kinds of control sequences may be more informative. If by "hierarchy" one simply means a symmetrical higher order rule configuration, then there are ways to discover if people perceive this. The best way relies upon sequences generated from several different higher order rules. If, for example, a pattern arises from the string of rules (relating successive chunks) $\mathbf{N}^{+1} \mathbf{N}^{+3} \mathbf{N}^{+1}$, it has a symmetrical arrangement of two different rules. A control sequence would merely rearrange these higher order rules into $\mathbf{N}^{+1} \mathbf{N}^{+1} \mathbf{N}^{+3}$. The former can be recursively rewritten as $\mathbf{N}^{4}\left(\mathbf{N}^{1}(\mathbf{X})\right)$, whereas the latter cannot. For example, using the diatonic alphabet of Figure 2b, a linear rule sequence such as

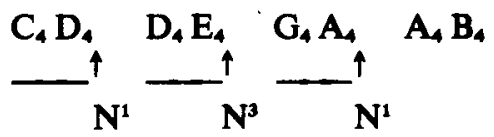

can also be expressed as $\mathbf{N}^{4}\left(\mathrm{~N}^{1}(C D)\right)$, whereas

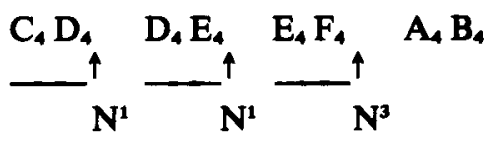

cannot be recursively rewritten. If people find sequences of the first kind (Expression 8) easier to reconstruct than those of the second, this is evidence in support of two things: (1) People respond to manipulations of higher order structure alone; and (2) people use the symmetry of hierarchical expressions to advantage in recall.

Deutsch's patterns unfortunately do not contain several different higher order rules. As indicated earlier, they are limited to repeated operations of a single rule. Nevertheless, nonrandom control sequences that are more informative than random ones can be devised. These would involve simply another, but nonsymmetrical, higher order rule arrangement based upon the same lower order rules. Consider, for example, Sequence 3 in Figure 4 which is based upon the $\mathrm{G}$ major scale. An appropriate control for this pattern would tamper with this higher order rule arrangement but not lower order ones (i.e., melodic chunks would be intact):

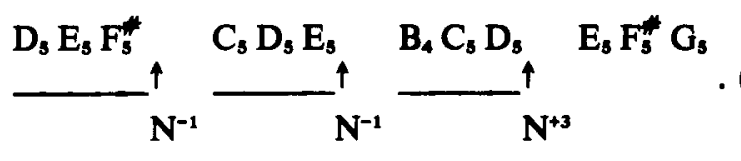

This sequence has equivalent lower order structure to Deutsch's Sequence 3, but it is not hierarchical. Again, if this pattern is harder to reconstruct than Sequence 3, then we have some support for the idea that superior performance with the hierarchical sequences does not derive simply from lower order rules. Furthermore, such differences again would show that people do respond to higher order structure and, in particular, that they find the configuration which can be recursively rewritten easier. Of course, there is the possibility that such a control sequence would turn out to be easier than its hierarchical counterpart, a happenstance that would suggest the reality of higher order rule configurations, but might also complicate life for the theorist who believes in hierarchical simplicity. But that, of course, is the point. We must pose realistic tests of hypotheses about higher order structure and risk being wrong.

Thus, any observed difference in performance on hierarchical patterns (e.g., Sequence 3) and appropriate controls (e.g., Expression 10) will give us evidence that people do respond to manipulations of higher order structure alone. But only if the former patterns are easier than the latter can we conclude that people somehow use the symmetrical rule arrangements of hierarchical expressions to advantage. Let me emphasize the "somehow" in the previous sentence. Such a difference does not necessarily tell us that people do, in fact, encode the hierarchical sequence recursively. It is quite possible that both sequences are perceived as linear structures, but that Sequence 3, with a linear rule string of $N^{1} N^{1} N^{1}$, is easier than its control of $\mathbf{N}^{-1} \mathbf{N}^{-1} \mathbf{N}^{+3}$.

These examples assume that a hierarchical sequence is one based upon rule symmetry. However, if by "hierarchy" one means not rule symmetry but simply a two-tiered set of melodic subsequences, then it is necessary to demonstrate the psychological reality of both tiers. If listeners use a higher level subsequence to encode a pattern, then manipulations of patterning within this level alone should influence performance. As we have seen, the higher order structure across the four patterned sequences a listener encountered in Deutsch's first study varied minimally 
relative to differences in lower order structure. A more convincing demonstration that higher order properties within a single sequence do matter would rely upon control sequences that rearrange melodic chunks as already described. For example, perhaps linearly encoded higher order subsequences such as $3 N^{1}$ (e.g., $D_{3} E_{5} F_{3}^{*} G_{8}$ for Sequence 3) are easier than controls such as $2 \mathrm{~N}^{-1}, \mathrm{~N}^{+3}$ (e.g., $\mathrm{F}_{8}^{*} \mathrm{E}_{\mathrm{s}} \mathrm{D}_{3} \mathrm{G}_{8}$, as in Expression 10) because they involve fewer rules. If so, then this manipulation would be successful in revealing that people are sensitive to higher order rule arrangements.

Finally, other controls may be required in addition to those which vary higher order structure. One important backup for inferences about specific rule representations is the contour control sequence. This is discussed in the next section.

To summarize, control sequences that tamper directly with higher order rule properties are more refined instruments for addressing questions about hierarchical structure than are random controls. The use of rule-based control sequences does not necessarily eliminate the need for other kinds of control sequences (e.g., contour controls). However, random sequences rarely inform about the relative contributions of lower and higher levels of structure in perception.

(2) Does rhythm affect the perception of any higher order structure? Deutsch concludes that temporal segmentation affects perceived structure. She characterizes perceived structure in terms of specific $\mathrm{N}^{ \pm j}$ rules that are parsed by a given rhythm. Unfortunately, this conclusion also runs aground on structural ambiguity on two counts. Deutsch fails to acknowledge, and hence control for, the fact that both melodic and temporal relations involve several correlated kinds of information. As a result, her studies do not eliminate plausible alternative hypotheses that have to do with the nature and timing of accents.

On the first count, as suggested earlier, inferences about encoding of specific musical intervals (i.e., $\mathrm{N}^{ \pm j}$ rules) can be drawn only when correlated effects of contour have been ruled out (Dowling \& Fujitani, 1971). That is, the rules Deutsch proposes enforce not only regularity of musical intervals but also regularity of contour. This is important for at least two reasons: (1) Contour changes can serve as accents and thereby identify the temporally regular onsets of rule subsequences in experimental patterns; and (2) correlated contours within rule-based patterns may, in themselves, contribute to the differential performance of listeners in various rhythmic conditions.

It appears, then, that control sequences are required that will gauge the contribution of pattern contour. Sequence 1 exhibits the regular contour of -+--+--+--+ , but the contour of the control pattern Deutsch used for this sequence was $\mathrm{o}-+\mathrm{o}++-++\mathrm{o}-$. Given the two arguments presented above, these sequences may produce performance differences merely because accents, namely contour changes, in the former mark the onset of simple one-rule subsequences but not in the latter. This argument differs from Deutsch's in emphasizing the role of contour and accent in detecting musical interval rules. But, according to the second point mentioned above, performance may also differ simply as a function of the difference in number and relative placement of contour changes. That is, in some tasks, listeners can identify patterns on the basis of contour alone. It is simple to devise control sequences that rule out this latter interpretation. For example, a control pattern with a contour equivalent to that of Sequence 1 and containing the same notes rearranged is:

$$
G_{3} C_{3}^{*} F_{3}^{*} D_{3} B_{4} \quad G_{3} D_{3} \quad G_{4} A_{4}^{*} G_{4} F_{4}^{*} B_{4}
$$

Patterns that control for correlated contour effects are useful in assessing the degree to which the differential performance of listeners in various rhythm conditions stems from highlighted contour invariances. This is because pauses that segment a pattern compatibly with respect to interval rule structure will also segment it compatibly with respect to contour. Thus, in Experiment 1, temporal segmentation of random controls showed complex contours with minimal constancies: $(0-)(+0)(++) \ldots$ over threetone groups. This same segmentation, however, not only preserves the temporal predictability of contour changes which mark rule onsets in the structured sequence, but (related to point 2 above) also highlights contour invariances over three-tone groups: $(-+)$ $(-+)(-+)(-+)$. Similarly, in Experiment 2 compatible segmentation preserves contour invariant properties and incompatible segmentation invariably destroys these properties. The moral here is that temporal segmentation may have its primary effects upon grouping by contour and not upon $\mathrm{N}^{ \pm \mathbf{j}}$ rules.

A second, and related, count on which Deutsch's conclusions run aground has to do with her inferences about time. Specifically, her inferences about temporal "parsing" neglect the fact that time relations have nominal (same or different period), ordinal (shorter or longer), interval (150 msec, 300 $\mathrm{msec} . .$.$) , and even ratio (300 \mathrm{msec} / 150 \mathrm{msec}=2$, etc.) properties. In contrast to her treatment of melodic relations, in which she infers perception of musical intervals but ignores ordinal pitch differences, in dealing with rhythm, Deutsch infers that ordinal time differences are critical but ignores the fact that she has varied metric time relations. To be specific, Deutsch argues that "pauses" "parse" a pattern. These words suggest that, regardless of actual time 
periods in her patterns, the effects she observed would hold. That is, "pause" and "parse" are indefinite with respect to the metrics of time intervals so that presumably pauses of $400 \mathrm{msec}$ or other arbitrarily selected values (or even variable pauses) would as effectively "parse" the sequence. While this may be the case, Deutsch's time rules are not arbitrarily selected. The temporally segmented patterns are carefully crafted to preserve good rhythmic form; in fact, Figure 3 portrays one of these rhythms. Consequently, these experiments do not eliminate a most obvious possibility, namely that metric time relations, which direct attention to accents, are responsible for her findings.

Finally, it is worth pointing out that the metric time hierarchy, shown in Figure 3, naturally identifies accent subsequences which correspond to notes in the higher order melodically generated subsequences of Deutsch. (In light of the foregoing discussion, these accents could well be distinguished by contour changes.) However, because she has ignored the implications of hierarchies of time rules and relative accent, Deutsch is forced to postulate hierarchies of melodic sequences instead. These observations, when taken together with the fact that each of Deutsch's patterns has a distinctively different contour, also create a plausible alternative explanation for her results. Compatible rhythms (1) identify initial tones within each melodic chunk as temporally related accents, and (2) facilitate the related percept of invariant contours [as in $(-+)$ ]. Incompatible rhythms produce different accent subsequences, ones associated with unreliable contour cues. Therefore, with compatible rhythms a skilled listener can easily use distinctive contours to intelligently guess about and catalogue patterns at the time of recall.

The main point of this section is that there are several kinds of relational structure that are correlated within serial patterns. Control sequences are extremely important in allowing a researcher to finely tune her examination to the desired kind of relationship. In the case of Deutsch's studies, she has failed to eliminate the possibility that rhythm, rather than facilitating the perception of the rules she proposes actually interacts with contour.

To conclude at a more general level, Deutsch's theoretical inferences appear unwarranted. Her conclusion that people perceive hierarchical structure is difficult to accept both because her definition of hierarchical structure is unclear and because she used inappropriate control sequences. And her assertion that rhythm facilitates encoding of interval rule structure fails to acknowledge the fact that other kinds of rules, in addition to those she proposes, may interact with rhythm.

I should emphasize that 1 do not argue about the truth or falsity of Deutsch's statements. Her state- ments may well prove to be true. In fact, I believe that people can, under certain circumstances, perceive hierarchical structure and that rhythmic patterning facilitates a top-down attentional strategy which encourages pickup of higher order melodic rules. I am simply arguing that experiments such as these do not allow us to draw such inferences. As a result, her findings should be viewed with caution.

\section{CONCLUSIONS}

Finally, the most important point about this analysis is not that we view with caution one or two published experiments. Rather, there are general lessons to be drawn about how to conduct meaningful research with serial patterns. These have to do with definitions of lower and higher order melodic and temporal rule structures and with criteria for designing control conditions in serial pattern research.

Real music is a complex blending of many kinds of relations with various sorts of higher order rule configurations. In bringing this part of our environment into the laboratory, we must inevitably simplify and catalogue. But it is quite important to be clear and consistent in our use of terms and conventions as well as in our choice of controls when we make this transition.

\section{REFERENCE NOTE}

1. Balzano, G. J. The structural uniqueness of the diatonic order. Paper presented at the Western Psychological Association, Symposium on Cognitive Structure of Musical Pitch, San Francisco, April 1978.

\section{REFERENCES}

Deutsch, D. The processing of structured and unstructured tonal sequences. Perception \& Psychophysics, 1980, 28, 381-389.

Dowling, W. J., \& Fujitani, D. S. Contour, interval, and pitch recognition in memory for melodies. Journal of the Acoustical Society of America, 1971, 49, 524-531.

Garner, W. R., \& Gotrwald, R. L. The perception and learning of temporal patterns. Quarterly Journal of Experimental Psychology, 1968, 20, 97-109.

Greeno, J. G., \& Simon, H. A. Processes for sequence production. Psychological Review, 1974, 81, 187-198.

HaHN, J., \& Jones, M. R. Invariance in auditory frequency relation. Scandinavian Journal of Psychology, 1981, 22, 1-16. Idson, W. L., \& MAssaro, D. W. A bidimensional model of pitch in the recognition of melodies. Perception \& Psychophysics, 1978, 24, 551-565.

JoNEs, M. R. Higher order organization in serial recall of digits. Journal of Experimental Psychology, 1973, 99, 106-119.

Jones, M. R. Cognitive representations of serial patterns. In B. Kantowitz (Ed.), Human information processing: Tutorials in performance cognition. Potomac, Md: Erlbaum, 1974.

Jones, M. R. Levels of structure in the reconstruction of temporal and spatial serial patterns. Journal of Experimental Psychology: Human Learning and Memory, 1976, 2, 475-488. (a)

Jones, M. R. Time, our lost dimension: Toward a new theory of 
perception, attention, and memory. Psychological Review, 1976, 83, 323-355. (b)

Jones, M. R. Auditory patterns: The perceiving organism. In E. C. Carterette \& M. P: Friedman (Eds.), Handbook of perception (Vol. 8). New York: Academic Press, 1978.

Jones, M. R. Only time can tell: An essay on the topology of mental space and time. Critical Inquiry, 1981, 7, 557-576.

Jones, M. R., \& BAIRD, J. J. Memory for symmetry: Real or artifact? American Journal of Psychology, 1979, 92, 627-651.

JoNe8, M. R., MAsen, D. J., \& KIDD, G. R. Rate and structure in memory for auditory patterns. Memory \& Cognition, 1978, 6, 246-258.

Jones, M. R., \& ZAmostry, K. P. Memory and rule structure in the prediction of serial patterns. Journal of Experimental Psychology: Human Learning and Memory, 1975, 104, 295-306.

KallmaN, H. J., \& MAssaro, D. W. Tone chroma is functional in melody recognition. Perception \& Psychophysics, 1979, 26, 32-36.

Kotovsky, K., \& Simon, H. A. Empirical tests of a theory of human acquisition of concepts for sequential patterns. Cognitive Psychology, 1973, 4, 399-424.

Martin, J. Rhythmic (hierarchical) vs. serial structure in speech and other behavior. Psychological Review, 1972, 79, 487-509.

Povel, D. Internal representation of simple temporal patterns. Journal of Experimental Psychology: Human Perception and Performance, 1981, 7, 3-18.

Restle, F. Theory of serial pattern learning: Structural trees. Psychological Review, 1970, 77, 481-495.

REstLe, F. Structural ambiguity in serial pattern learning. Cognitive Psychology, 1976, 8, 357-381.

Restle, F., \& Brown, E. R. Organization of serial pattern learning. In G. H. Bower (Ed.), The psychology of learning and motivation: Advances in research and theory (Vol. 4). New York: Academic Press, 1970.
Simon, H. A. Complexity and the representation of patterned sequences of symbols. Psychological Review, 1972, 79, 369-382.

Simon, H. A., \& Kotovsky, K. Human acquisition of concepts for sequential patterns. Psychological Review, 1963, 70, 534-546.

Simon, H. A., \& Sumner, R. K. Pattern in music. In B. Kleinmuntz (Ed.), Formal representation of human judgment. New York: Wiley, 1968.

TulviNG, E. Memory research: What kind of progress? In L. Nilsson (Ed.), Perspectives on memory research: Essays in honor of Uppsala University's 500th anniversary. Hillsdale, N.J: Erlbaum, 1979.

\section{NOTES}

1. The musical notation, $C_{4} D_{4}$, etc., used throughout the manuscript refers to musical notes within common musical scales where the subscript refers to the particular octave array (i.e., $C_{4}$ is middle $C$ and $A_{4}$ is $440 \mathrm{~Hz}$ ).

2. This property assists in formalizing the fact that various inversions of a chord are often treated as equivalent (see, e.g., examples in Figure 2b).

3. Often a whole linear pattern such as this, if repeated without break, will become a hierarchical one. This presents an interesting and unexplored case for the study of subjective reorganization (Garner \& Gottwald, 1968).

4. Postulation of time symmetries raises profound philosophical and scientific questions, questions which remain unsolved. Nevertheless, their possible relevance to psychology opens up exciting new ways of conceiving of familiar problems.

(Manuscript received March 26, 1981; revision accepted for publication August 3, 1981.) 